\title{
Chronic Post Traumatic Headaches Unresponsive to Medication Management
}

Hasoon $\mathrm{J}^{1^{\star}}$, Berger $\mathrm{A}^{1}$

${ }^{1}$ Beth Israel Deaconess Medical Center, Department of Anesthesia, Critical Care, and Pain Medicine, Harvard Medical School, Boston, MA, USA

Corresponding Author: Jamal Hasoon MD

Address: Beth Israel Deaconess Medical Center, Department of Anesthesia, Critical Care, and Pain Medicine, Harvard Medical School, Boston, MA, USA; E-mail: Jhasoon@bidmc.harvard.edu

Received date: 15 April 2020; Accepted date: 02 May 2020; Published date: 12 May 2020

Citation: Hasoon J, Berger A. Chronic Post Traumatic Headaches Unresponsive to Medication Management. Asp Biomed Clin Case Rep. 2020 May 12;3(2):114-115.

Copyright (C) 2020 Hasoon J, Berger A. This is an open-access article distributed under the Creative Commons Attribution License, which permits unrestricted use, distribution, and reproduction in any medium provided the original work is properly cited.

\section{Keywords}

Headache, Post Traumatic Headache, Traumatic Brain Injury, Chronic Pain

Chronic post traumatic headaches (PTH) are being more frequently seen in military medicine due to the increasing prevalence of patients suffering from explosive related head injuries (1). Data from recent wars in the Middle East have shown a higher proportion of traumatic brain injuries (TBI) caused by blast-related and explosive injuries (1-3). Patients who have served in military combat operations in the middle-east have a higher chance to develop PTH related to TBIs. These patients can be difficult to manage as there are limited medication options for the treatment of these headaches in patients. This brief report describes 2 patients who suffered from chronic PTH who failed a multitude of medication management.

Both patients were middle aged male patients who were suffering from chronic PTH. The first patient had a history of a mild TBI from being in the military while the other patient had a history of PTH after a head injury during a physical fight. Both patients described chronic daily headaches. Both headaches were primarily occipital and temporal in nature. These headaches were not associated with photophobia or nausea. The first patient rated his headaches at $8 / 10$ severity while the second patient rated his at $6 / 10$ on a numerical rating scale. These patients had been evaluated by primary care physicians as well as neurologists before being referred to pain management and headache specialists at our clinic.

Both patients were trialed on a variety of medications as well as physical therapy. The patients tried acetaminophen, NSAIDs, and gabapentin. The first patient had also been trialed on tricyclic antidepressants and Topiramate but was unable to tolerate the sedating side effects. The second patient was also trialed on beta-blockers as well as antiepileptic medications, though without much relief and issues with cognition. Neither patient was able to achieve optimal relief with medication management. Both required referral for interventional pain management procedures.

Chronic PTH appears to be especially challenging to treat. We attempted a variety of medication management options, excluding opioids, in our 


\section{Short Communication}

patients without success. Neither patient was able to achieve satisfactory relief with medication management for their headaches, and also experienced unwanted side effects including sedation and issues with cognition. There is some evidence that interventional procedures can be a valuable tool for headache disorders such as third occipital neuralgia and cervicogenic headaches (4-6). Physicians may want to consider referring these patients for interventional therapies in the future.

\section{References}

[1] Owens BD, Kragh JF Jr, Wenke JC, Macaitis J, Wade CE, Holcomb JB. Combat wounds in operation Iraqi Freedom and operation Enduring Freedom. J Trauma. 2008 Feb;64(2):295-99. [PMID: 18301189]

[2] Wojcik BE, Stein CR, Bagg K, Humphrey RJ, Orosco J. Traumatic brain injury hospitalizations of U.S. army soldiers deployed to Afghanistan and Iraq. Am J Prev Med. 2010 Jan;38(1 Suppl):S108-16. [PMID: 20117583]

[3] Hasoon J. Blast-associated traumatic brain injury in the military as a potential trigger for dementia and chronic traumatic encephalopathy. US Army Med Dep J. 2017 Jan-Jun;(1-17):102-105. [PMID: 28511279]

[4] Zhou L, Hud-Shakoor Z, Hennessey C, Ashkenazi A. Upper cervical facet joint and spinal rami blocks for the treatment of cervicogenic headache. Headache. 2010 Apr;50(4):657-63. [PMID: 20132336]

[5] Hasoon J, Berger AA. Radiofrequency neurotomy for long-term relief of third occipital neuralgia. Saudi J Anaesth. 2020 Apr-Jun;14(2):266-67. [PMID: 32317894]

[6] Govind J, King W, Bailey B, Bogduk N. Radiofrequency neurotomy for the treatment of third occipital headache. J Neurol Neurosurg Psychiatry. 2003 Jan;74(1):88-93. [PMID: 12486273] 